\title{
Improvement in High-fat Diet-induced Obesity and Insulin Resistance upon Uptake of PS-B1, a Fermented Product Prepared from Soy Flour Using Lactic Acid Bacteria
}

\author{
Kyoshiro Yamaguchi*, Marie Iwanaga-Suehiro, Kyoko Fujimoto, Masashi Fukasawa, \\ Ryuzo Sakakibara
}

Department of Biochemistry, Faculty of Pharmaceutical Sciences, Nagasaki International University, Sasebo, Nagasaki, Japan

Email address:

kyamaguchi@niu.ac.jp (K. Yamaguchi)

${ }^{*}$ Corresponding author

To cite this article:

Kyoshiro Yamaguchi, Marie Iwanaga-Suehiro, Kyoko Fujimoto, Masashi Fukasawa, Ryuzo Sakakibara. Improvement in High-fat Diet-induced Obesity and Insulin Resistance upon Uptake of PS-B1, a Fermented Product Prepared from Soy Flour Using Lactic Acid Bacteria. Journal of Food and Nutrition Sciences. Vol. 8, No. 4, 2020, pp. 81-88. doi: 10.11648/j.jfns.20200804.12

Received: June 3, 2020; Accepted: June 19, 2020; Published: July 4, 2020

\begin{abstract}
PS-B1 is a fermented product prepared from soy flour using lactic acid bacteria. Over a 10-week period, C57BL/6J mice were reared under laboratory conditions on a normal diet (control, $n=5)$, high-fat diet $(H F, n=5)$, or high-fat diet supplemented with 4\% PS-B1 (HF-P, n=6). After 10 weeks, the change in weight gain, intestinal and epididymal fat accumulation, serum and liver biochemical parameters, and gene expression in the mice was investigated. HF diet-induced weight gain and increase in intestinal and epididymal fat accumulation were lower in mice fed with HF-P diet than in mice fed with HF diet, suggesting that PS-B1 prevented HF diet-induced obesity in HF-P mice. Furthermore, the levels of liver lipids (triglycerides, TG; non-esterified fatty acid, NEFA; total cholesterol, TC), serum TC, serum glucose, and serum insulin were significantly increased in the HF group than those in control mice. In HF-P mice, neither serum TC nor serum glucose levels were reduced. In contrast, the levels of liver lipids and serum insulin were lower in HF-P mice than in HF mice, suggesting that PS-B1 reduced these parameters in HF-P mice. The homeostatic model assessment of insulin resistance (HOMA-IR) value, which was calculated from the serum glucose and insulin levels, was $21.5 \pm 4.2$ in the HF mice. However, the HOMA-IR $(8.2 \pm 0.2)$ values were significantly decreased in the HF-P mice, suggesting that PS-B1 improves insulin resistance. Additionally, we compared the expression levels of stearoyl-CoA desaturase-1 $(S c d 1)$ in the liver. Quantitative RT-PCR showed increased expression of $S c d 1$ in HF mice compared to that in control mice. Furthermore, ingestion of PS-B1 led to reduced expression of Scd1 mRNA in HF-P mice, implying that PS-B1 is effective in reducing the expression of the gene encoding SCD1. These results suggest that the anti-obesity effect of PS-B1 and improvement in fat accumulation upon PS-B1 uptake may be due to improvement in insulin resistance and reduction in the expression level of $S c d 1$.
\end{abstract}

Keywords: Lactic Acid Bacteria, Soy Flour, Biogenics, Lipid Metabolism, Obesity Prevention

\section{Introduction}

Lifestyle-related diseases is the generic term for diseases associated with individuals' way of life, including hypertension, dyslipidemia, and diabetes. These diseases are triggered by certain lifestyles, such as eating habits and exercise habits, which reduce healthy life expectancy. In addition, as a lifestyle-related disease progresses, complications occur, leading to decrease in quality of life. For this reason, many health foods and supplements have been developed, which are sold as primary preventive measures. Probiotics [1] are one of the health supplements that show a preventive effect on lifestyle-related diseases [2-4]. Recently, attention has been focused on the usefulness of biogenics for the prevention and improvement of lifestyle-related diseases [5-7]. Biogenics are defined as "food ingredients that have a bioregulatory function directly or through intestinal flora" [8]. Fermentation products of lactic acid bacteria are one such product. Obesity is well known to lead to lifestyle-related diseases. Biogenics are expected to contain substances 
effective for prevention of obesity and could have several applications in medicine. We have been studying the applications of PS-B1, a product fermented by lactic acid bacteria. PS-B1 is a fermented product obtained by co-culturing 21 types of human intestinal bacterial strains selected to mimic the intestinal environment of healthy individuals $[9,10]$. Soy flour is used for fermentation. PS-B1 has been selected for research to clarify the usefulness of biogenics at the biochemical level [11-13]. In a previous study, we examined the effect of PS-B1 uptake on high-fat (HF) diet-fed mice models of nonalcoholic steatohepatitis (STAM mice). The results suggested that PS-B1 decreased the weight of liver and reduced the levels of TG in the plasma and liver in HF diet-fed STAM mice [14]. These findings suggest that PS-B1 prevents steatohepatitis in vivo. In addition, we examined the effects of PS-B1 uptake in long-term high-sucrose diet-fed mice. It was observed that PS-B1 uptake reduced weight gain, serum cholesterol levels, serum insulin levels, and expression of lipogenic enzymes and insulin receptor substrate [15]. In general, obesity is well known to cause insulin resistance [16]. It is natural that disturbances in dietary habits promote the appearance of insulin resistance. In this study, the effects of dietary administration of PS-B1 on wild-type mice administered with a HF diet were investigated.

\author{
First stage \\ Regeneration of \\ Second stage \\ Culture in 10 groups \\ Third stage \\ Small-scale culture \\ Fourth stage \\ Large-scale culture
}

16 lactic acid bacteria and 5 bifidobacterium
16 lactic acid bacteria and 5 bifidobacterium (MRS broth)

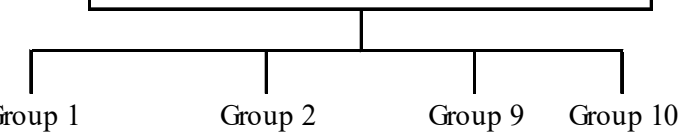

Group 1
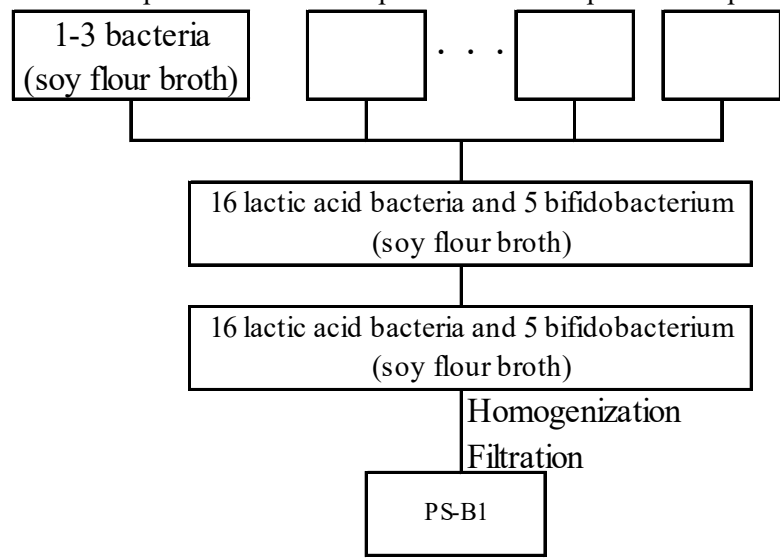

Figure 1. Preparation of PS-B1. PS-B1 was prepared as a fermented product of soy flour by a four-step complex culture method using 16 indigenous lactic acid bacteria and 5 indigenous bifidobacteria. Lactic acid bacteria and bifidobacteria used for the preparation of PS-B1 and their distribution in the secondary cultures are shown in Table 1.

\section{Materials and Methods}

\subsection{Preparation of PS-B1}

Soy flour medium was prepared by adding pesticide-free, organically cultivated soy flour, glucose, yeast extract, skim milk powder, and defoaming agent to water, followed by homogenization. PS-B1, a fermented filtrate obtained by culturing 21 types of lactic acid bacteria in soy flour medium, was prepared according to the process shown in Figure 1 by BIOGENOMICS Co., Ltd., (Nagasaki, Japan) [9, 10]. In the first stage of fermentation, all 21 strains of bacteria were cultured separately. In the second stage of fermentation, 10 sets of bacteria consisting of two to three strains each, as listed in Table 1, were co-cultured. In the third and fourth stage of fermentation, all the bacteria were co-cultured and filtrated, resulting in the formation of PS-B1. The average weight of lyophilized powder obtained from $100 \mathrm{~mL}$ of PS-B1 was $4.35 \mathrm{~g}$.

Table 1. Sixteen lactic acid bacteria and five bifidobacteria used for the preparation of PS-B1 and their grouping in secondary cultures.

\begin{tabular}{llll}
\hline Groups & Lactic acid bacteria & & \\
\hline 1 & Bifidobacterium longum subsp. longum & Enterococcus faecium & Enterococcus durans \\
2 & Lactobacillus paracasei subsp. paracasei & Lactobacillus delbrueckii subsp. bulgaricus & - \\
3 & Enterococcus faecium & Lactobacillus brevis & Lactobacillus plantarum \\
4 & Enterococcus faecium & Bifidobacterium bifidum & Bifidobacterium breve \\
5 & Bifidobacterium adolescentis & Lactobacillus acidphilus & Bifidobacterium longum subsp. infantis \\
6 & Enterococcus durans & Lactobacillus gasseri & Lactobacillus salivarius \\
7 & Lactobacillus gasseri & Enterococcus faecium & Lactobacillus rhamnosus \\
8 & Enterococcus faecalis & Lactobacillus delbrueckii subsp. delbrueckii & Lactobacillus helveticus \\
9 & Lactobacillus casei & Bifidobacterium adolescentis & Lactococcus lactis subsp. lactis \\
10 & Enterococcus faecium & Enterococcus durans & Lactococcus lactis subsp. cremoris \\
\hline
\end{tabular}




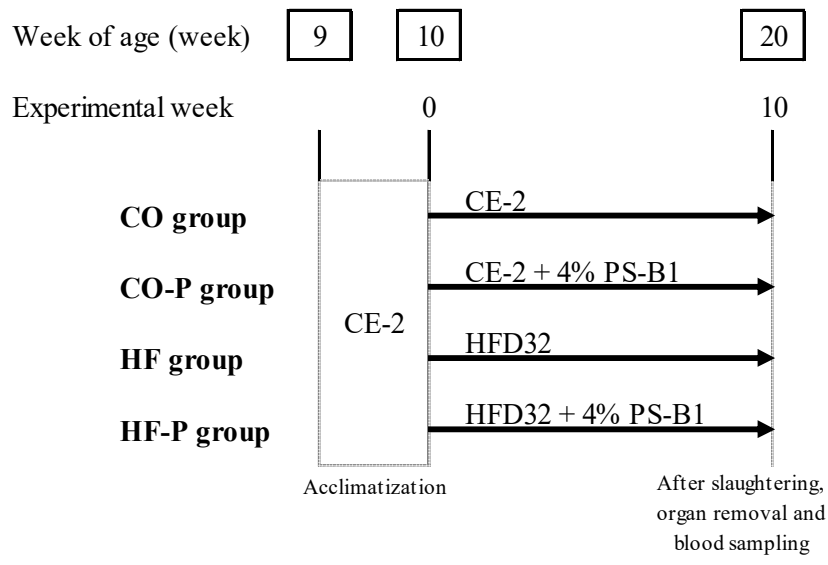

Figure 2. Diet plan for experimental animals. Nine-weeks-old C57BL/6J male mice were randomly divided into four groups ( $n=5$ or 6 per group) after a week of acclimatization. The mice in the $\mathrm{CO}$ (control) and $\mathrm{CO}-\mathrm{P}$ groups were fed with CE-2 solid diet and CE-2 diet supplemented with $4 \%$ lyophilized PS-B1, respectively. The mice in the HF and HF-P groups were fed with HFD32 (high-fat diet) solid diet and HFD32 diet supplemented with 4\% lyophilized PS-B1, respectively.

\subsection{Animals}

Animal experiments were conducted in accordance with the Standard Relating to the Care and Management of Laboratory Animals and Relief of Pain (Notice no. 88, Ministry of the Environment, Government of Japan), and the protocols for animal experiments were approved by the Ethics Review Committee of Nagasaki International University (approval number 141). The diet plan of the experimental animals is shown in Figure 2. Nine-weeks-old male C57BL/6J mice (CLEA Japan, Inc., Tokyo, Japan) were housed in a room with controlled temperature, relative humidity, and light and dark cycle $(22 \pm$ $2{ }^{\circ} \mathrm{C}, 60 \pm 2 \%$ relative humidity, and light from 7:00 to 19:00 h, respectively). The mice had free access to water and a normal diet (CE-2, CLEA Japan, Inc., Tokyo, Japan). The mice were acclimatized for one week. Later, half the number of mice were switched to a 10-week HF diet (HFD32, CLEA Japan, Inc., Tokyo, Japan) containing $32.0 \%$ crude fat $(56.7 \%$ fat kcal ( $\%)$ ), while the remaining mice were fed a normal diet containing $4.6 \%$ crude fat $(12.2 \%$ fat $\mathrm{kcal}(\%))$. The diet of half the number of mice in each group was supplemented with $4 \%$ PS-B1 (Table 2). This resulted in four groups with five or six mice each: $\mathrm{CE}-2$ (control, $\mathrm{n}=5$ ), CE-2 + 4\% PS-B1 (CO-P, $\mathrm{n}=5)$, HFD32 (HF, $\mathrm{n}=5$ ), and HFD32 + 4\% PS-B1 (HF-P, $\mathrm{n}=6$ ). Body weight and food intake were recorded twice and thrice a week, respectively, for 10 weeks. After the 10-week experimental period, the mice were denied access to food and water overnight and were then sacrificed using isoflurane inhalation anesthetic. The liver, epididymal fat, and intestinal fat were then dissected, weighed, and stored at $-80^{\circ} \mathrm{C}$. Blood was collected from the heart, followed by separation of serum, which was stored at $-80^{\circ} \mathrm{C}$.

Table 2. Nutrients in experimental diets.

\begin{tabular}{lllll}
\hline Ingredient & CE-2 & $\begin{array}{l}\text { CE-2 } \\
\mathbf{4 \%} \text { PS-B1 }\end{array}$ & HFD32 \\
\hline Moisture (\%) & 8.8 & - & 6.2 & HFD32 \\
Crude protein (\%) & 25.5 & 25.6 & 25.5 & - \\
Crude fat (\%) & 4.6 & 4.4 & 32.0 & 25.6 \\
Crude fiber (\%) & 5.1 & - & 2.9 & 30.7 \\
Crude ash (\%) & 7.0 & 7.5 & 4.0 & - \\
Nitrogen-free extract (\%) & 48.6 & 50.1 & 29.4 & 5.6 \\
Energy (kcal/100g) & 339.1 & 343.5 & 507.6 & 51.6 \\
Fat kcal (\%) & 12.2 & 11.5 & 56.7 & 505.3 \\
\hline
\end{tabular}

\subsection{Biochemical Analysis of Serum}

The serum biochemical parameters, including TG, NEFA, $\mathrm{TC}$, and glucose levels, were assayed using LabAssay ${ }^{\mathrm{TM}}$ kit series (FUJIFILM Wako Pure Chemical Corporation, Osaka, Japan). Insulin was assayed using a Mouse Insulin Measurement Kit (Morinaga Institute of Biological Science,
Inc., Kanagawa, Japan).

\subsection{HOMA-IR Value}

The HOMA-IR values were calculated according to the method described by Matthews et al. [17] and Hosojima et al. [18] and mentioned as follows:

HOMA-IR $=$ fasting serum insulin levels $(\mu \mathrm{U} / \mathrm{mL}) \times$ fasting serum glucose levels $(\mathrm{mg} / \mathrm{dL}) / 405$

\subsection{Liver Lipid Analysis}

Total lipid was extracted from the liver by Folch method [19]. The extract was dissolved in 2-propanol. The TG, NEFA, and TC levels were assayed using LabAssay ${ }^{\mathrm{TM}}$ kit series.

\subsection{Quantitative RT-PCR}

Total RNA was isolated from the mouse liver tissue using
TRIZOL Reagent (Life Technologies Japan Ltd., Tokyo, Japan) according to the manufacturer's protocol. Quantitative RT-PCR assays were performed using the PikoReal Real-Time PCR System (Thermo Fisher Scientific K. K., Tokyo, Japan). Briefly, $500 \mathrm{ng}$ of total RNA was reverse transcribed into cDNA using the ReverTra Ace qPCR RT Master Mix (TOYOBO CO., LTD., Osaka, Japan) according to the manufacturer's protocol to quantify the mRNA levels of sterol regulatory element binding protein 1c (Srebp-1c), 
diacylglycerol acyltransferase 2 (Dgat2), fatty acid synthase (Fas), and Scdl. Each amplification mixture contained $2 \mathrm{ng}$ of cDNA, appropriate concentration of the forward and reverse primers (Table 3), and THUNDERBIRD SYBR qPCR Mix (TOYOBO CO., LTD., Osaka, Japan). The PCR cycling parameters were $95^{\circ} \mathrm{C}$ for $1 \mathrm{~min}$, and 40 cycles of $95^{\circ} \mathrm{C}$ for $15 \mathrm{~s}$ and $60^{\circ} \mathrm{C}$ for $1 \mathrm{~min}$. Relative expression was calculated using the comparative relative standard curve method [20]. We used acidic ribosomal phosphoprotein P0 (36B4) mRNA as the relative control [21] for our study.

Table 3. Primer pairs used for Scd1, Srebp-1c, Fas, Dgat2, and $36 B 4$ in the present study.

\begin{tabular}{lll}
\hline Gene symbol & & Sequence (5'-3') \\
\hline \multirow{2}{*}{ Scd1 } & Forward & GAGGCCTGTACGGGATCATA \\
& Reverse & CAGCCGAGCCTTGTAAGTTC \\
Srebp-1c & Forward & ATCTCCTAGAGCGAGCGTTG \\
& Reverse & TATTTAGCAACTGCAGATATCCAAG \\
Fas & Forward & CCCTTGATGAAGAGGGATCA \\
& Reverse & GAACAAGGCGTTAGGGTTGA \\
Dgat 2 & Forward & AGGCCCTATTTGGCTACGTT \\
\multirow{2}{*}{36 B 4} & Reverse & CATCAGGTACTCGCGAAGC \\
& Forward & GAGGAATCAGATGAGGATATGGGA \\
& Reverse & AAGCAGGCTGACTTGGTTGC \\
\hline
\end{tabular}

Scd1, stearoyl-CoA desaturase 1; Srebp-1c, sterol regulatory element binding protein 1c; Fas, fatty acid synthase; Dgat2, diacylglycerol acyltransferase 2; 36B4, acidic ribosomal phosphoprotein P0.

\subsection{Statistical Analysis}

Each value is presented as mean \pm SE. The differences among the experimental groups were analyzed using unpaired t-test, followed by Bonferroni's post hoc test; $p<$ 0.05 was considered statistically significant. Means with the same letter are not significantly different from each other.

\section{Results}

\subsection{Influence of PS-B1 on the Body Weight of Mice}

Male C57BL/6J mice were fed with control, CO-P, HF, or
HF-P diet. After 10 weeks, the increase body weight was larger in HF mice than in control mice. Although dietary bulk per week was not different between HF and HF-P mice (data not shown), the increase in HF diet-induced body weight was less in HF-P mice than in HF mice (Figure 3).

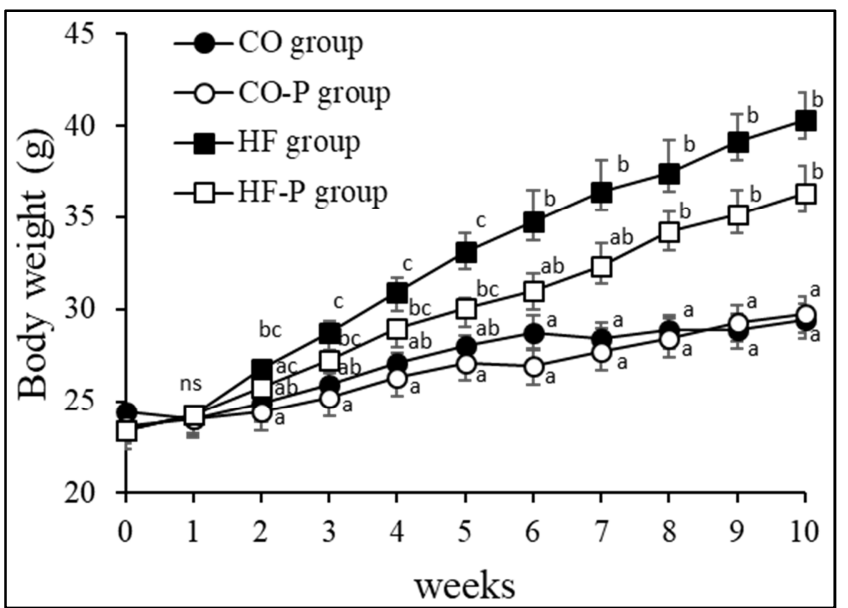

Figure 3. Body weight of mice fed with CE-2 or HFD32 diet with or without $4 \%$ of PS-B1. Body weight of C57BL/6J male mice in the CO group (•), CO-P group (०), HF group (ロ) and HF-P group ( $\square$ ) was measured twice a week for 10 weeks. All values are presented as mean $\pm S E$. Means with the same letter are not significantly different from each other. The differences among the experimental groups were analyzed using unpaired t-test, followed by Bonferroni's post hoc test; $p<0.05$ was considered statistically significant.

\subsection{Influence of PS-B1 on Intestinal Fat, Epididymal Fat, and Weight of Liver in Mice}

After 10 weeks of feeding, there was an increase in intestinal and epididymal fat in HF-fed mice compared to that in control mice. However, HF diet-induced increase in intestinal and epididymal fat was lower in HF-P mice than in HF mice (Table 4). No difference in liver weight was observed between mice under different feeding conditions (Table 4).

Table 4. Weights of tissues of mice fed with different diets.

\begin{tabular}{|c|c|c|c|c|}
\hline & CO group & CO-P group & HF group & HF-P group \\
\hline Intestinal fat (g) & $0.14 \pm 0.02^{\mathrm{ab}}$ & $0.09 \pm 0.01^{\mathrm{a}}$ & $0.94 \pm 0.11^{\mathrm{c}}$ & $0.53 \pm 0.08^{b c}$ \\
\hline Epididymal fat (g) & $0.24 \pm 0.03^{\mathrm{ab}}$ & $0.16 \pm 0.01^{\mathrm{a}}$ & $1.72 \pm 0.16^{\mathrm{c}}$ & $1.32 \pm 0.23^{\mathrm{bc}}$ \\
\hline Liver weight (g) & $1.13 \pm 0.04^{\mathrm{a}}$ & $1.17 \pm 0.06^{\mathrm{a}}$ & $1.37 \pm 0.08^{\mathrm{a}}$ & $1.32 \pm 0.09^{\mathrm{a}}$ \\
\hline
\end{tabular}

The weight of intestinal fat, epididymal fat, and liver in mice fed with different diets was measured for 10 weeks. All values are presented as mean \pm SE. Means with the same letter are not significantly different from each other. The differences among the experimental groups were analyzed using unpaired t-test, followed by Bonferroni's post hoc test; $\mathrm{p}<0.05$ was considered statistically significant.

\subsection{Influence of PS-B1 on Serum and Liver Biochemical Parameters in Mice}

After 10 weeks of feeding, the serum TC, serum insulin, serum glucose, and liver lipid levels (TG, NEFA, and TC) were found to be higher in HF mice than in control mice. However, the increase in $\mathrm{HF}$ diet-induced serum insulin levels and liver lipids was lower in HF-P mice than in HF mice (Tables 5, 6). The serum insulin levels were lower in HF-P mice than in HF mice, indicating that HOMA-IR was improved upon ingestion of PS-B1, further indicating improvement in insulin resistance upon ingestion of PS-B1 (Table 7). Since some factors have been reported to cause insulin resistance due to obesity [16], we also examined these factors. Liver NEFA levels were 1.94 times higher in HF mice than in control mice; contrastingly, the levels were only 1.31 times higher in HF-P mice than in control mice, 
indicating that PS-B1 ingestion reduced the increase in liver NEFA levels by 33\%. Furthermore, liver TC levels were 1.97 times higher in HF mice than in control mice; contrastingly, they were only 1.41 times higher in HF-P mice, indicating that PS-B1 ingestion reduced the increase in liver TC levels by $29 \%$ (Table 6$)$.

Table 5. Serum biochemical markers in mice fed with different diets.

\begin{tabular}{|c|c|c|c|c|}
\hline & CO group & CO-P group & HF group & HF-P group \\
\hline Serum TG (mg/dL) & $58.8 \pm 3.4^{\mathrm{a}}$ & $62.4 \pm 3.5^{\mathrm{a}}$ & $70.1 \pm 6.1^{\mathrm{a}}$ & $65.0 \pm 3.3^{\mathrm{a}}$ \\
\hline Serum NEFA (mEq/L) & $0.97 \pm 0.02^{\mathrm{a}}$ & $0.97 \pm 0.07^{\mathrm{a}}$ & $0.96 \pm 0.06^{\mathrm{a}}$ & $0.80 \pm 0.04^{\mathrm{a}}$ \\
\hline Serum TC $(\mathrm{mg} / \mathrm{dL})$ & $64.5 \pm 2.0^{\mathrm{a}}$ & $52.2 \pm 4.0^{\mathrm{a}}$ & $117.3 \pm 5.7^{\mathrm{b}}$ & $111.0 \pm 12.1^{\mathrm{b}}$ \\
\hline Serum Insulin (ng/mL) & $0.36 \pm 0.02^{\mathrm{a}}$ & $0.45 \pm 0.05^{\mathrm{a}}$ & $1.27 \pm 0.29^{\mathrm{a}}$ & $0.68 \pm 0.15^{\mathrm{a}}$ \\
\hline Serum Glucose (mg/dL) & $98.2 \pm 5.6^{\mathrm{a}}$ & $118.2 \pm 12.0^{\mathrm{ab}}$ & $226.1 \pm 28.7^{\mathrm{bc}}$ & $242.2 \pm 4.5^{\mathrm{c}}$ \\
\hline
\end{tabular}

Serum TG, NEFA, TC, insulin, and glucose levels were measured in mice fed with different diets for 10 weeks. All values are presented as mean \pm SE. Means with the same letter are not significantly different from each other. The differences among the experimental groups were analyzed using unpaired t-test, followed by Bonferroni's post hoc test; $\mathrm{p}<0.05$ was considered statistically significant.

Table 6. Liver biochemical markers in mice fed with different diets.

\begin{tabular}{llll}
\hline & CO group & CO-P group & HF group \\
\hline Liver TG $(\mathrm{mg} / \mathrm{g})$ & $17.59 \pm 1.84^{\mathrm{a}}$ & $15.71 \pm 2.33^{\mathrm{a}}$ & $23.94 \pm 1.62^{\mathrm{a}}$ \\
Liver NEFA $(\mu \mathrm{mol} / \mathrm{g})$ & $9.50 \pm 1.38^{\mathrm{a}}$ & $6.40 \pm 0.78^{\mathrm{a}}$ & $18.47 \pm 1.47^{\mathrm{b}}$ \\
Liver TC $(\mathrm{mg} / \mathrm{g})$ & $1.42 \pm 0.22^{\mathrm{a}}$ & $1.20 \pm 0.16^{\mathrm{a}}$ & $2.80 \pm 0.24^{\mathrm{b}}$ \\
\hline
\end{tabular}

Liver TG, NEFA, and TC levels were measured in mice fed with different diets for 10 weeks. All values are presented as mean \pm SE. Means with the same letter are not significantly different from each other. The differences among the experimental groups were analyzed using unpaired t-test, followed by Bonferroni's post hoc test; $\mathrm{p}<$ 0.05 was considered statistically significant.

Table 7. HOMA-IR calculated for mice fed with different diets.

\begin{tabular}{ll}
\hline & HOMA-IR \\
\hline CO group & $2.24 \pm 0.13^{\mathrm{a}}$ \\
CO-P group & $3.54 \pm 0.71^{\mathrm{a}}$ \\
HF group & $21.51 \pm 4.22^{\mathrm{b}}$ \\
HF-P group & $8.21 \pm 0.20^{\mathrm{b}}$ \\
\hline
\end{tabular}

HOMA-IR values were calculated for mice reared under different dietary conditions. All values are presented as mean \pm SE. Means with the same letter are not significantly different from each other. The differences among the experimental groups were analyzed using unpaired t-test, followed by Bonferroni's post hoc test; $p<0.05$ was considered statistically significant.

\subsection{Influence of PS-B1 on Lipid Synthetic Gene Expression in Mice}

The expression of Fas, Dgat2, Srebp-1c, and Scd1 was higher in HF mice than in control mice. There was no difference in the expression levels of Fas, Dgat2, and Srebp-1c between HF-P mice and HF mice (Table 8). In contrast, ingestion of PS-B1 reduced $S c d 1$ expression in HF-P mice compared to that in HF mice, implying that PS-B1 is effective in reducing the expression of the gene encoding SCD1.

Table 8. Expression level of mRNA in the liver of mice fed with different diets.

\begin{tabular}{llll}
\hline Gene symbol & CO group & CO-P group & HF group \\
\hline Scd1 & $1.00 \pm 0.07^{\mathrm{a}}$ & $0.84 \pm 0.04^{\mathrm{b}}$ & $4.24 \pm 0.35^{\mathrm{c}}$ \\
Srebp-1c & $1.00 \pm 0.12^{\mathrm{a}}$ & $1.46 \pm 0.19^{\mathrm{a}}$ & $7.43 \pm 1.23^{\mathrm{b}}$ \\
Fas & $1.00 \pm 0.14^{\mathrm{a}}$ & $1.37 \pm 0.28^{\mathrm{a}}$ & $3.56 \pm 0.42^{\mathrm{b}}$ \\
Dgat2 & $1.00 \pm 0.07^{\mathrm{a}}$ & $1.11 \pm 0.03^{\mathrm{a}}$ & $1.96 \pm 0.13^{\mathrm{a}}$ \\
\hline
\end{tabular}

The mRNA levels of Scd1, Srebp-1c, Fas, and Dgat2 were measured in mice fed with different diets for 10 weeks. Each value was normalized to the value of $36 B 4$ mRNA, which was used as an internal standard, and expressed as a relative mRNA level to control group. All values are presented as mean \pm SE. Means with the same letter are not significantly different from each other. The differences among the experimental groups were analyzed using unpaired t-test, followed by Bonferroni's post hoc test; $p<0.05$ was considered statistically significant.

\section{Discussion}

PS-B1 is a "biogenics" [8] containing various fermentation metabolites. It is produced by fermenting soy flour using a traditional method, which involves 21 different species of intestinal bacteria. In our previous study, we found evidence that PS-B1 may effectively inhibit the growth of cancer cell lines [11], suppress allergic reactions in rats [9], promote hair growth in mice [13], and improve bowel movements and skin conditions in humans [10]. Other studies have reported that 
probiotics prevent weight gain in HF diet-induced obesity and thus improve metabolic syndrome [22-24]. It is highly possible that PS-B1, which is a fermented product of intestinal bacteria, exhibits a similar effect by itself or by activating the intestinal bacterial flora of the host. In this study, using wild-type mice, we examined the effects of PS-B1 ingestion on HF diet-induced obesity and adverse metabolic consequences of a HF diet. Over a 10-week period, C57BL/6J mice were reared under laboratory conditions on normal (control), HF, or HF-P diet. After 10 weeks, diet-induced weight gain and increase in intestinal and epididymal fat accumulation were lower in HF-P mice than in HF mice, suggesting that PS-B1 prevented HF diet-induced obesity in HF-P mice (Figure 3, Table 4).

The levels of intestinal and epididymal fat were investigated in mice. Intestinal fat levels were 6.71 times higher in HF mice than in control mice; however, they were only 3.79 times higher in HF-P mice than in control mice, indicating that PS-B1 ingestion reduced the increase in intestinal fat levels by $44 \%$. Epididymal fat levels were 7.17 times higher in HF mice than in control mice, whereas, they were only 5.50 times higher in HF-P mice than in control mice, indicating that PS-B1 ingestion reduced the increase in epididymal fat levels by $23 \%$ (Table 4 ). These findings suggested that PS-B1 reduced intestinal and epididymal fat accumulation. The possible mechanism of this effect could be that PS-B1 inhibits lipid absorption and synthesis, promotes metabolic processes such as beta-oxidation, or promotes excretion. In a previous study, we examined the effects of PS-B1 ingestion in long-term high-sucrose diet-fed mice, and observed that PS-B1 ingestion reduced the expression of lipogenic enzymes such as acetyl-CoA carboxylase $(A c c)$ and Fas [15]. Based on these observations, in the present study, we compared the expression levels of Fas, Dgat2, and Scd1. HF mice exhibited increased expression of Fas, Dgat2, and Scd1. There was no decrease in the expression levels of Fas and Dgat2 in HF-P mice (Table 8). In contrast, HF mice exhibited increased expression of $S c d 1$, while PS-B1 uptake reduced the expression of $S c d 1$ in HF-P mice, indicating that PS-B1 is effective in reducing the expression of the gene encoding SCD1. In a study on SCD1, Ntambi et al. found that among mice fed a $\mathrm{HF}$ diet, $\mathrm{SCD}^{-/-}$mice exhibited less weight gain than $\mathrm{SCD}^{+/+}$mice. This indicated that $\mathrm{SCD} 1^{-/}$upregulates lipid oxidation genes and downregulates lipid synthesis genes. They concluded that the increased oxidation of saturated fats in $\mathrm{SCD}^{-/}$mice was the result of metabolic activity characterized by a higher rate of beta-oxidation [25]. In the present study, PS-B1 ingestion reduced HF diet-induced obesity-associated increase in liver lipid levels in HF mice (Table 6). Thus, the reduced accumulation of intestinal and epididymal fat in HF-P mice might have been a consequence of reduced $S c d 1$ expression caused by PB-S1 uptake, which then facilitated beta-oxidation.

Obesity is associated with insulin resistance, and numerous studies have demonstrated that a HF diet leads to insulin resistance in mice. Biddinger et al. reported that mice fed a HF diet developed worse steatosis and exhibited a greater increment in LDL cholesterol and insulin levels than mice fed a low-fat diet [26]. These findings correspond to those of the present study, wherein we found that 10 weeks of HF diet increased serum glucose and insulin levels, thereby inducing insulin resistance, as determined by a high HOMA-IR value. In contrast, HF-P mice exhibited improved HOMA-IR due to lower serum insulin levels than HF mice (Table 7). Therefore, it was suggested that PS-B1 is effective in improving insulin resistance. A previous study reported that $\mathrm{SCD} 1^{-/-}$mice fed a $\mathrm{HF}$ diet gained less weight and exhibited greater insulin signaling and glycogen accumulation in brown adipose tissue than $\mathrm{SCD}^{+/+}$mice $[25,27]$. Furthermore, the mice administered with antisense oligonucleotide (ASO), as a treatment to reduce SCD1 expression, exhibited reduced levels of glucose productivity, gluconeogenesis, and glycogenolysis [28]. Thus, the researchers of that study concluded that reduced SCD1 expression ameliorated insulin resistance. From the results of glucose, insulin, and pyruvate tolerance tests performed on mice bred under the same conditions as in the present study, we found that PS-B1 enhances insulin activity (data not shown). The current findings suggest that PS-B1 reduces insulin resistance by reducing the expression of $S c d 1$.

Transcription of SCD1 is regulated by SREBPs. Biddinger et al. reported that mice fed a HF diet exhibited increased expression of the transcription factors $S r e b p-1 c$ and $S c d 1$ [26]. In this study, we observed that PS-B1 ingestion reduced $S c d 1$ expression, but did not affect the expression of Srebp-1c. Additionally, the expression of Fas, which, similar to $S c d 1$, is regulated by $S r e b p-1 c$, was not affected. These observations offer further insights into the mechanism of action of PS-B1 in reducing obesity. Presumably, a transcription factor other than SREBP-1c could reduce the expression of $S c d 1$, leading to reduced obesity and insulin resistance. Xiao et al. examined the effects of expression of B-cell translocation gene 1 (Btgl) on obesity in $d b / d b$ mice, which are used as a genetic model of obesity. Despite reduced levels of Btgl mRNA and protein, $B t g 1$ overexpression reduced hepatic steatosis in $d b / d b$ mice, while Btgl knockdown induced hepatic steatosis in wild-type mice. They also reported that Btgl overexpression reduced the activity of activating transcription factor 4 (ATF4), thereby preventing the expression of $S c d 1$ [29]. Furthermore, in vitro analyses using HepG2 cells and mouse primary hepatocytes indicated that Btgl overexpression improved insulin signaling, while Btg1 knockdown worsened it. In addition, Btg1 overexpression reduced insulin resistance in both $d b / d b$ and wild-type mice. The same study also reported that mice with Btg1 overexpression were resistant to insulin resistance, which was induced by the high-carbohydrate diet [30]. Thus, to further elucidate the mechanism by which PS-B1 improves obesity and insulin resistance, the role of upstream transcription factors in reducing the expression of $S c d 1$ needs to be investigated.

\section{Conclusion}

PS-B1 is a "biogenics" containing various fermentation 
metabolites. In this study, we found that PS-B1 contains factors that can reduce the expression of $S c d 1$ and PS-B1 consumption may improve obesity and insulin resistance. We expect that PS-B1 will become a useful food as a "biogenics" suitable for preventive medicine.

\section{Acknowledgements}

The authors gratefully acknowledge the work of past and present members (E. Ide, Y. Tasaki, A. Ikeda, and Y. Eguchi) of our laboratory. In this study, we received PS-B1 and research funding from BIOGENOMICS Co., Ltd. We would like to thank Editage (www.editage.com) for English language editing.

\section{References}

[1] Fuller R., "Probiotics in man and animals," J. appl. bacteriol., 66, 365-378, 1989.

[2] Wong V. W., Wong G. L., Chim A. M., Chu W. C., Yeung D. K., Li K. C., and Chan H. L., "Treatment of nonalcoholic steatohepatitis with probiotics. A proof-of-concept study," Ann Hepatol., 12, 256-262, 2013.

[3] Sun J., and Buys N. J., "Glucose- and glycaemic factor-lowering effects of probiotics on diabetes: a meta-analysis of randomised placebo-controlled trials," $\mathrm{Br}$. $J$. Nutr., 115, 1167-1177, 2016.

[4] Huang R., Wang K., and Hu J., "Effect of probiotics on depression: A systematic review and meta-analysis of randomized controlled trials," Nutrients., 8, 483, 2016, doi: $10.3390 /$ nu8080483.

[5] Takahashi T., Masuda Y., Yoshida K., and Mizuno M., "Development of fermented rice drink using lactic acid bacteria isolated from kimoto," Seibutsu-kogaku., 94, 63-69, 2016.

[6] Aoi W., Iwasa M., and Abe R., "Investigation of fermented milk-derived biogenics that improve exercise-induced glucose metabolism in middle-aged to elderly people," Descente sports science., 38, 164-171, 2017.

[7] Odashiro K., Fukata M., Saito K., Wakana C., Maruyama T., Sasuga Y., Fukui M., and Fujino T., "The effects of lactic acid bacteria-fermented soymilk extract on patients with colonic polyps: a randomized, double-blind, placebo-controlled pilot trial," J. Integr. Stud. Diet. Habits., 25, 20-25, 2014.

[8] Mitsuoka T., "Significance of dietary modulation of intestinal flora and intestinal environment," Bioscience Microflora., 19, $15-25,2000$.

[9] Nodake Y., Miura R., Ryoya H., Momii R., Toda S., and Sakakibara R., "Improvement of lipid metabolism and ovalbumin-induced type I allergy by use of soybean milk fermented by 16 indigenous lactic acid bacteria," J. Food Nutr. Sci., 4, 113-119, 2016, doi: 10.11648/j.jfns.20160404.17.

[10] Yamaguchi K., Matsumoto S., Fuchigami T., Tsuchida M., Honda H., and Sakakibara R., "Effect of PS-B1 (a fermented product cultured from soy flour using lactic acid bacteria) on defecation and skin condition," J. Integr. Stud. Diet. Habits., 30, 111-122, 2020.
[11] Nodake Y., Ogasawara M., Honda H., Fukasawa M., and Sakakibara R., "Characterization and preliminary purification of the anti-cancer substance (s) in the fermented products cultivated from soybean milk using lactic acid bacteria," Saito Ho-on kai Mus. Nat. Hist., Res. Bull., 73, 17-21, 2008.

[12] Nodake Y., Fukasawa M., Arakawa M., and Sakakibara R., "Effect of the fermented product cultivated from soybean milk using lactic acid bacteria, PS-B1, on liver function and lipid metabolism," J. Integr. Stud. Diet. Habits., 22, 13-19, 2011.

[13] Sakakibara R., Kawatsu R., Honda H., Toda S., Yamaguchi K., and Nodake Y., "Effect of PS-B1 (a fermented product cultured from soybean milk using lactic acid bacteria) on hair growth in vivo. (1st report)," J Jpn Hair Sci Assoc., 51, 147-154, 2019.

[14] Fukasawa M., Nodake Y., Kawatsu R., Honda H., Yamaguchi K., and Sakakibara R., "In vivo efficacy evaluation of the fermented product cultivated from soybean milk using lactic acid bacteria, PS-B1, in STEM ${ }^{\mathrm{TM}}$ model of nonalcoholic steatohepatitis," unpublished.

[15] Toda S., Nodake Y., and Sakakibara R., "Effect of PS-B1 (a fermented product cultured from soybean milk using lactic acid bacteria) on metabolic disturbances due to excessive sucrose intake," Proceedings of The 3rd Int. Conf. on Pharma and Food, p. 124, 2016.

[16] Vogeser M., König D., Frey I., Predel H., Parhofer K. G., and Berg A., "Fasting serum insulin and the homeostasis model of insulin resistance (HOMA-IR) in the monitoring of lifestyle interventions in obese persons," Clin. Biochem., 40, 964-968, 2007.

[17] Matthews D. R., Hosker J. P., Rudenski A. S., Naylor B. A., Treacher D. F., and Turner R. C., "Homeostasis model assessment: insulin resistance and $\beta$-cell function from fasting plasma glucose and insulin concentrations in man," Diabetologia., 28, 412-419, 1985.

[18] Hosojima H., and Uchida K., "A practical clinical index of insulin resistance in obese patients with impaired glucose tolerance -An application of HOMA's index-," J. Japan Diab. Soc., 41, 433-441, 1998.

[19] Folch J., Lees M., and Sloane Stanley G. H., "A simple method for the isolation and purification of total lipides from animal tissues," J. Biol. Chem., 226, 497-509, 1957.

[20] Larionov A., Krause A., and Miller W., "A standard curve based method for relative real time PCR data processing," BMC Bioinform., 6, 2005, doi: 10.1186/1471-2105-6-62.

[21] Sawada T., Miyoshi H., Shimada K., Suzuki A., Okamatsu-Ogura Y., Perfield II J. W., Kondo T., Nagai S., Shimizu C., Yoshioka N., Greenberg A. S., Kimura K., and Koike T., "Perilipin overexpression in white adipose tissue induces a brown fat-like phenotype," PLoS One., 5, e14006, 2010, doi: 10.1371/journal.pone.0014006.

[22] Ma X., Hua J., and Li Z., "Probiotics improve high fat diet-induced hepatic steatosis and insulin resistance by increasing hepatic NKT cells," J. Hepatol., 49, 821-830, 2008, doi: 10.1016/j.jhep.2008.05.025.

[23] Wang J., Tang H., Zhang C., Zhao Y., Derrien M., Rocher E., van-Hylckama Vlieg J. E. T., Strissel K., Zhao L., Obin M., and Shen J., "Modulation of gut microbiota during probiotic-mediated attenuation of metabolic syndrome in high fat diet-fed mice," The ISME Journal., 9, 1-15, 2015. 
[24] Kim B., Kwon J., Kim M-S., Park H., Ji Y., Holzapfel W., and Hyun C-K., "Protective effects of Bacillus probiotics against high-fat diet-induced metabolic disorders in mice," PLoS One., 13, e0210120, 2018, doi: 10.1371/journal.pone.0210120.

[25] Ntambi J. M., Miyazaki M., Stoehr J. P., Lan H., Kendziorski C M., Yandell B. S., Song Y., Cohen P., Friedman J. M., and Attie A. D., "Loss of stearoyl-CoA desaturase-1 function protects mice against adiposity," Proc. Natl. Acad. Sci. U.S.A., 99, 11482-11486, 2002.

[26] Biddinger S. B., Almind K., Miyazaki M., Kokkotou E., Ntambi J. M., and Kahn C. R., "Effects of diet and genetic background on sterol regulatory element-binding protein-1c, stearoyl-CoA desaturase 1 , and the development of the metabolic syndrome," Diabetes., 54, 1314-1323, 2005.

[27] Rahman S. M., Dobrzyn A., Lee S-H., Dobrzyn P., Miyazaki M., and Ntambi J. M., "Stearoyl-CoA desaturase 1 deficiency increases insulin signaling and glycogen accumulation in brown adipose tissue," Am. J. Physiol. Endocrinol. Metab., 288, E381-E387, 2005, doi: 10.1152/ajpendo.00314.2004.

[28] Gutiérrez-Juárez R., Pocai A., Mulas C., Ono H., Bhanot S., Monia B. P., and Rossetti L., "Critical role of stearoyl-CoA desaturase-1 (SCD1) in the onset of diet-induced hepatic insulin resistance," J. Clin. Invest., 116, 1686-1695, 2006, doi: 10.1172/JCI26991.

[29] Xiao F., Deng J., Guo Y., Niu Y., Yuan F., Yu J., Chen S., and Guo F., "BTG1 ameliorates liver steatosis by decreasing stearoly-CoA desaturase 1 (SCD1) abundance and altering hepatic lipid metabolism," Sci. Signal., 9, ra50, 1-11, 2016.

[30] Xiao F., Deng J., Yu J., Guo Y., Chen S., and Guo F., “A novel function of B-cell translocation gene 1 (BTG1) in the regulation of hepatic insulin sensitivity in mice via c-Jun," The FASEB Journal., 30, 348-359, 2016. 